\title{
Imaging in anatomy: a comparison of imaging techniques in embalmed human cadavers
}

\author{
Grit Gesine Ruth Schramek ${ }^{1 *}$, Dietrich Stoevesandt ${ }^{2}$, Ansgar Reising $^{3}$, Jan Thomas Kielstein ${ }^{3}$, Marcus Hiss ${ }^{3+}$ \\ and Heike Kielstein ${ }^{1+}$
}

\begin{abstract}
Background: A large variety of imaging techniques is an integral part of modern medicine. Introducing radiological imaging techniques into the dissection course serves as a basis for improved learning of anatomy and multidisciplinary learning in pre-clinical medical education.

Methods: Four different imaging techniques (ultrasound, radiography, computed tomography, and magnetic resonance imaging) were performed in embalmed human body donors to analyse possibilities and limitations of the respective techniques in this peculiar setting.

Results: The quality of ultrasound and radiography images was poor, images of computed tomography and magnetic resonance imaging were of good quality.

Conclusion: Computed tomography and magnetic resonance imaging have a superior image quality in comparison to ultrasound and radiography and offer suitable methods for imaging embalmed human cadavers as a valuable addition to the dissection course.
\end{abstract}

Keywords: Medical education, Teaching, Ultrasound, Magnetic resonance imaging, Computed tomography, X-ray, Imaging

\section{Background}

A wide variety of imaging techniques is an integral part of modern medicine [1], as is the knowledge about indications, side effects and limitations of techniques spanning from bed-side ultrasound over high resolution computed tomography to functional magnetic resonance imaging. Over the last decades medical students increasingly learn to interpret different imaging techniques in the first years of medical school as part of the anatomical curriculum. Cornerstones of this curriculum are the 'living anatomy' course on the one hand, and medical images and PBL (Problem-Based-Learning) on the other hand [2-4]. Introducing radiological imaging techniques into the dissection course and macroscopic anatomy classes enhances students' long-term ability to identify anatomical structures in medical images [5-7]. Realizing this, we introduced medical images to our education in

\footnotetext{
* Correspondence: ruth.schramek@medizin.uni-halle.de

${ }^{\dagger}$ Equal contributors

'Department of Anatomy and Cell Biology, Martin Luther University of Halle-Wittenberg, Magdeburger Str. 8, 06108 Halle (Saale), Germany Full list of author information is available at the end of the article
}

gross anatomy several years ago. So far, we are able to provide head and hip, knee and shoulder joint $\mathrm{CT}$ and MRI scans of our body donors, and use archived images for x-ray based techniques as well as sonography in volunteers (fellow students) in line with the 'living anatomy' courses. While the later one is not associated with safety hazards of the scanning itself, the potential discovery of pathological conditions needing further investigation or the need to obtain an informed consent, have to be considered as critical limitations [1]. Another fact is that images of young, healthy students do not have a lot in common with the bodies of the usually elderly body donors or future patients. There are age-related changes, physiological varieties and pathologies, and we have already been able to witness that students' fascination and motivation is increased by viewing changes of 'their' body donor on medical images and exploring them by dissection afterwards. Therefore, we are convinced that the preparation of structures that underwent imaging just prior to anatomical dissection causes an even greater gain in knowledge than the approach mentioned above.

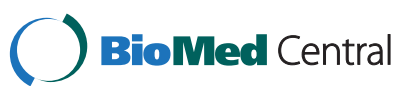


However, commonly used embalming fluid, containing small amounts of formalin, not only causes a significant swelling of soft tissues $[4,8]$, but also produces artifacts, that severely deteriorate image quality [9]. Due to the controversial discussion on imaging techniques, the aim of the present study was to compare four imaging techniques in a structured approach to decide which would be suitable for the different visualization needs of embalmed human donors and could thereby offer a valuable addition to the dissection course.

\section{Methods}

The study was approved by the ethics commission of the medical faculty of the Martin Luther University HalleWittenberg. Written informed consent for scientific investigations in general is given by all body donors prior to death at the Department of Anatomy and Cell Biology.

\section{Subjects}

For the study, we examined 23 human donors (11 male, 12 female; median age 81.1 years, SD 11.7 years, range 45 years). The cadavers investigated were preserved after standard embalming techniques, using a solution containing ethanol (77\%), unbuffered formalin, glycerine, and distilled water $(\sim 7 \%$, respectively). The procedure includes intravascular embalming for 8 hours, embalming in a solution bath for six to eight weeks, and storage at $2-4^{\circ} \mathrm{C}$ for up to two years. The following imaging techniques have been conducted:

\section{Ultrasound}

Renal size parameters, including renal length, width, and cortical thickness, of both kidneys of six body donors (3 male, 3 female) were evaluated by two independent and experienced sonographers. Cadavers were investigated lying in prone position with a CX 50 Ultrasound System, C5-1 Broadband Curved Array Transducer with PureWave technology, Philips, Hamburg, Germany.

\section{Radiography}

Both an anteroposterior (AP) and lateral view of the lumbar spine and pelvis have been performed with six body donors ( 3 male, 3 female) to assess the lumbar vertebral bodies and the infrarenal part of the aorta. Cadavers were investigated in supine position with the Mobilett+, Siemens, Erlangen, Germany. Evaluation has been carried out by an experienced radiologist.

\section{Computed tomography}

A whole-body scan, including head, thorax, abdomen, pelvis, shoulder and knee joints, has been performed with 12 body donors ( 5 male, 7 female). The system used was the Sensation64, Siemens, Erlangen, Germany. CTimages were acquired with $120 \mathrm{kV}$ and 500 effmAs and primarily reconstructed in $0.6 \mathrm{~mm}$ slices $(0.5 \mathrm{~mm}$ increment). Afterwards multi planar reconstructions were made in axial, sagittal and coronal orientation $(5 \mathrm{~mm}$ slice thickness). Evaluation has been carried out by an experienced radiologist.

\section{Magnetic resonance imaging}

Comparable to the CT-scan a whole-body scan has been taken from 4 human donors (1 male, 3 female) with the Skyra 3 T, Siemens, Erlangen, Germany. MRI images for whole body imaging were acquired as axial T1-TSE (slice thickness $6 \mathrm{~mm}$, TR $680 \mathrm{~ms}$, TE $12 \mathrm{~ms}$ ), axial T2-TSE (slice thickness $4 \mathrm{~mm}$, TR $7870 \mathrm{~ms}$, TE $81 \mathrm{~ms}$ ), coronal T1-TSE (slice thickness $3 \mathrm{~mm}$, TR $903 \mathrm{~ms}$, TE $21 \mathrm{~ms}$ ) and sagittal T2-TSE images (slice thickness $4 \mathrm{~mm}$, TR $8520 \mathrm{~ms}$, TE $100 \mathrm{~ms}$ ). MRI images for knee joint were acquired as PD-weighted TSE sequences in axial, sagittal and coronal orientation (slice thickness $2.5 \mathrm{~mm}$, TR $2800 \mathrm{~ms}$, TE $19 \mathrm{~ms}$ ). Evaluation has been carried out by an experienced radiologist.

\section{Evaluation criteria}

The four clinical authors established imaging criteria for the explored imaging techniques according to the AIUM Practice Guideline for the Performance of an Ultrasound Examination [10], the European Guidelines on Quality Criteria for Diagnostic Radiographic Images [11], and the European Guidelines on Quality Criteria for Computed Tomography [12] (Tables 1, 2, 3 and 4). Five experienced sonographers and five experienced radiologists

Table 1 Evaluation of ultrasound images

\begin{tabular}{|c|c|c|c|c|c|c|c|}
\hline \multirow[t]{2}{*}{ Image quality criteria for ultrasound - kidneys } & \multicolumn{5}{|c|}{ Rating } & \multirow[t]{2}{*}{ Mean } & \multirow[t]{2}{*}{ Range } \\
\hline & Rater 1 & Rater 2 & Rater 3 & Rater 4 & Rater 5 & & \\
\hline 1. Visualization of long-axis length (both poles visible) & 1 & 1 & 0 & 0 & 1 & 0.6 & 1 \\
\hline 2. Visualization of transverse length & 1 & 0 & 0 & 1 & 0 & 0.4 & 1 \\
\hline 3. Visualization of renal cortex & 1 & 1 & 1 & 1 & 1 & 1 & 0 \\
\hline 4. Visualization of renal pelvis & 1 & 1 & 1 & 1 & 1 & 1 & 0 \\
\hline Total raw score & 4 & 3 & 2 & 3 & 3 & 3 & 2 \\
\hline Calculated score & 1 & 0.75 & 0.5 & 0.75 & 0.75 & 0.75 & 0.5 \\
\hline
\end{tabular}

Rating: $0=$ not visible, 1 = poorly visible, 2 = easily visible. 
Table 2 Evaluation of $x$-ray images

\begin{tabular}{|c|c|c|c|c|c|c|c|}
\hline \multirow{2}{*}{$\begin{array}{l}\text { Image quality criteria for x-ray - lumbar spine } \\
\text { and pelvis }\end{array}$} & \multicolumn{5}{|c|}{ Rating } & \multirow[t]{2}{*}{ Mean } & \multirow[t]{2}{*}{ Range } \\
\hline & Rater 1 & Rater 2 & Rater 3 & Rater 4 & Rater 5 & & \\
\hline 1. Visualization of the upper and lower plate surfaces & 1 & 1 & 1 & 1 & 1 & 1 & 0 \\
\hline 2. Visualization of the cortex and trabecular structures & 1 & 0 & 1 & 1 & 1 & 0.8 & 1 \\
\hline 3. Visualization of the aortic shape/course & 0 & 0 & 1 & 0 & 1 & 0.4 & 1 \\
\hline 4. Visualization of aortic calcifications & 2 & 1 & 1 & 1 & 1 & 1.2 & 1 \\
\hline Total raw score & 4 & 2 & 4 & 3 & 4 & 3.4 & 2 \\
\hline Calculated score & 1 & 0.5 & 1 & 0.75 & 1 & 0.85 & 0.5 \\
\hline
\end{tabular}

Rating: $0=$ not visible, $1=$ poorly visible, $2=$ easily visible.

rated the taken images. The sonographers evaluated ultrasound images, the radiologist rated $\mathrm{x}$-ray, CT, and MRI images. Evaluation of the images has been carried out individually by each expert without knowledge of the other raters' evaluation. Following De Crop et al. [13] and Benkhadra et al. [14], who have evaluated clinical images in Thiel's embalmed cadavers before, the criteria were rated with " 0 " if the anatomical structure was not visible, with " 1 " if the structure was poorly visible, and with " 2 " if the anatomical structure was easily visible. The sum of scores (total raw score) has then been divided by the number of criteria used for each imaging technique to get comparable values (calculated score).

In addition to the image quality criteria we evaluated the possibility of 3D reconstruction as well as the aspect of overall feasibility, including costs and logistics, for the four conducted imaging techniques.

\section{Results}

Ultrasound

The imaging quality of the examined kidneys was very poor (Figure 1). Although offering a non-invasive technique, severe intravascular gas artifacts, up to the small renal blood vessels (arcuate arteries) and in the surrounding tissue, were found (Additional file 1). Furthermore, in supine position some cadavers showed laminar subepidermal accumulations of gas. Tissue compression with the ultrasound transducer did not improve the imaging. The evaluation of reliable measurements of the kidneys was predominantly unsuccessful (Table 1).

\section{Radiography}

The quality of the obtained images was poor (Table 2). The sagging of abdominal organs and additional gas artefacts in the abdominal aorta worsened the imaging quality. In AP views the lumbar spine and pelvic bones were poorly detectable, in lateral views scarcely any structures were identifiable (Figure 2).

\section{Computed tomography}

The imaging quality of the skeletal system was very good (Figure 3). The scanning of other tissues was limited by subcutaneous, intravascular and intramedullary gas artefacts as well as the lack of contrast. Therefore the lungs and other parenchymal organs were scarcely assessable. The rating of the image quality criteria for $\mathrm{CT}$ is presented in Table 3.

\section{Magnetic resonance imaging}

The quality of joint images was very good even though this imaging technique was limited by an altered signal behaviour, especially of the subcutaneous tissue (Figure 4). Table 4 shows the evaluation results for the MRI images.

Table 3 Evaluation of CT images

\begin{tabular}{|c|c|c|c|c|c|c|c|}
\hline \multirow[t]{2}{*}{ Image quality criteria for CT - whole body } & \multicolumn{5}{|c|}{ Rating } & \multirow[t]{2}{*}{ Mean } & \multirow[t]{2}{*}{ Range } \\
\hline & Rater 1 & Rater 2 & Rater 3 & Rater 4 & Rater 5 & & \\
\hline 1. Visualization of thoracic wall & 2 & 2 & 2 & 2 & 2 & 2 & 0 \\
\hline 2. Visualization of entire lung parenchyma & 1 & 2 & 2 & 2 & 2 & 1.8 & 1 \\
\hline 3. Visualization of heart, aorta, and vena cava & 2 & 1 & 2 & 1 & 2 & 1.6 & 1 \\
\hline 4. Visualization of parenchymal organs & 1 & 2 & 2 & 2 & 2 & 1.8 & 1 \\
\hline 5. Visualization of vertebral structures & 2 & 2 & 2 & 2 & 2 & 2 & 0 \\
\hline 6. Visualization of shoulder, hip, and knee joint & 2 & 1 & 1 & 1 & 1 & 1.2 & 1 \\
\hline Total raw score & 10 & 10 & 11 & 10 & 11 & 10.4 & 1 \\
\hline Calculated score & 1.7 & 1.7 & 1.8 & 1.7 & 1.8 & 1.74 & 0.1 \\
\hline
\end{tabular}

Rating: $0=$ not visible, $1=$ poorly visible, $2=$ easily visible. 
Table 4 Evaluation of MRI images

\begin{tabular}{|c|c|c|c|c|c|c|c|}
\hline \multirow{2}{*}{$\begin{array}{l}\text { Image quality criteria for MRI - whole } \\
\text { body }\end{array}$} & \multicolumn{5}{|c|}{ Rating } & \multirow[t]{2}{*}{ Mean } & \multirow[t]{2}{*}{ Range } \\
\hline & Rater 1 & Rater 2 & Rater 3 & Rater 4 & Rater 5 & & \\
\hline 1. Visualization of thoracic wall & 2 & 2 & 2 & 2 & 2 & 2 & 0 \\
\hline 2. Visualization of entire lung parenchyma & 1 & 1 & 1 & 1 & 1 & 1 & 0 \\
\hline 3. Visualization of heart, aorta, and vena cava & 2 & 1 & 2 & 2 & 2 & 1.8 & 1 \\
\hline 4. Visualization of parenchymal organs & 2 & 2 & 2 & 2 & 2 & 2 & 0 \\
\hline 5. Visualization of vertebral structures & 1 & 2 & 2 & 2 & 2 & 1.8 & 1 \\
\hline 6. Visualization of shoulder, hip, and knee joint & 2 & 2 & 2 & 2 & 2 & 2 & 0 \\
\hline Total raw score & 10 & 10 & 11 & 11 & 11 & 10.6 & 1 \\
\hline Calculated score & 1.7 & 1.7 & 1.8 & 1.8 & 1.8 & 1.76 & 0.1 \\
\hline
\end{tabular}

Rating: $0=$ not visible, $1=$ poorly visible, 2 = easily visible.

In addition to the image quality criteria we evaluated the possibility of 3D reconstruction as well as the aspect of overall feasibility, including costs and logistics, for the four conducted imaging techniques. Results are shown in Table 5.

\section{Discussion}

The positive effects of introducing radiological images into the dissection course have already been described by several authors. Not only it enhances the students' learning effects in anatomy but it also increases the overall understanding of medical imaging, thus improving the students' long-term ability to identify anatomical structures both in vivo and in medical images [5-7,9,15]. Based on these encouraging reports, we have introduced medical images to our education in gross anatomy several years ago. So far, we are using ultrasound examinations in line with the 'living anatomy' seminars and provide head and hip, knee and shoulder joint CT and MRI scans of our body donors. Based on our own experiences, we agree with the findings of Chew et al. [9] and Bohl et al. [15] that combining post-

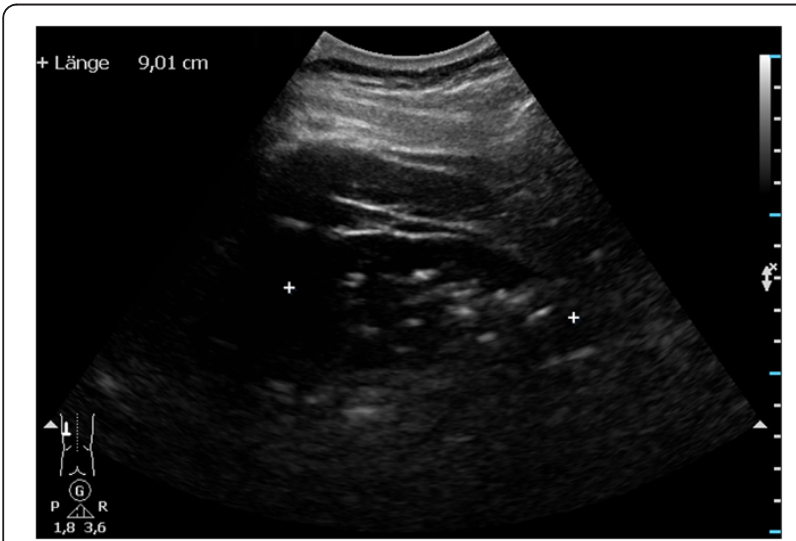

Figure 1 Very poor quality of ultrasound image of the kidney. The evaluation of reliable measurements of the kidney was unsuccessful due to severe gas artifacts. mortem radiological images and anatomical dissection of the same body donor leads to a greater gain in knowledge than using real patient images that are not comparable to the dissected cadavers in a one-to-one manner.

To our knowledge this is the first study comparing several imaging techniques of routine clinical use, i.e. ultrasound, radiography, computed tomography and magnetic resonance imaging, in embalmed cadavers. CT and MRI have been identified as techniques resulting in the best image quality, although logistics and price of imaging exceeds ultrasound or plain X-ray.

\section{Ultrasound}

Ultrasound, a routinely used bedside technique in clinical medicine, is not suitable to scan intraabdominal

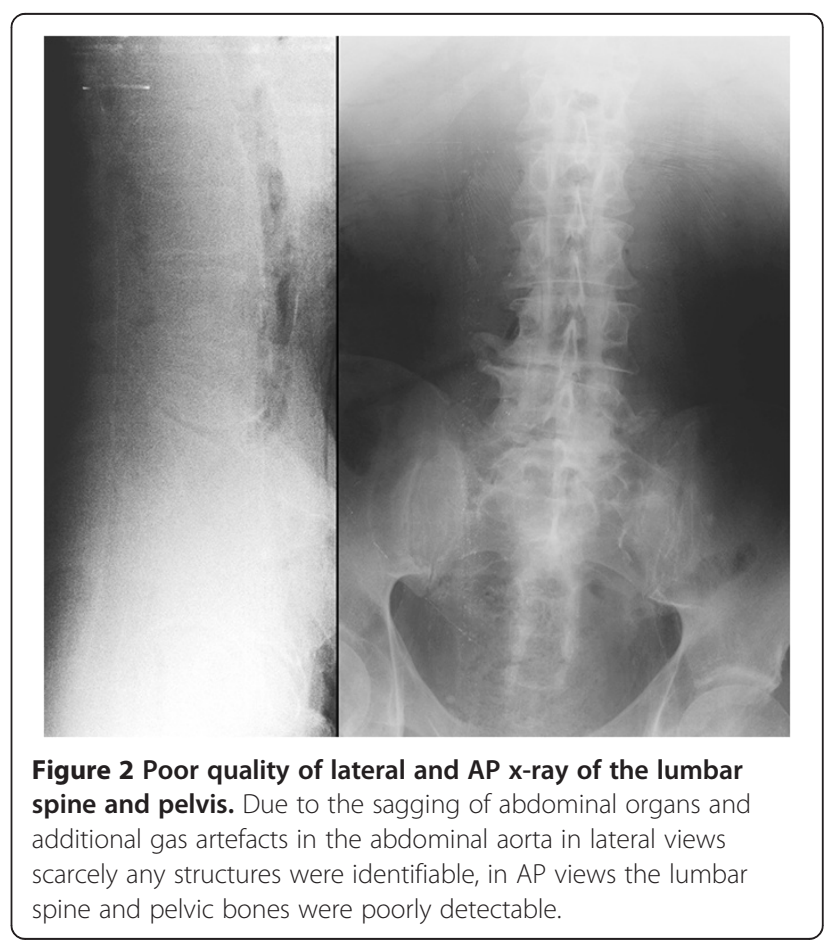




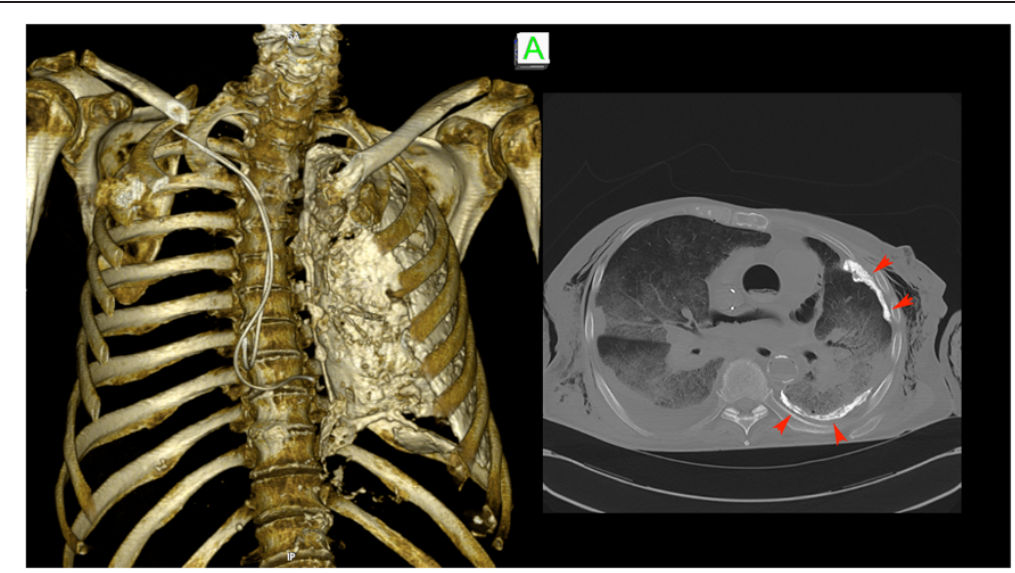

Figure 3 The CT imaging quality of the skeletal system was good. With the help of 3-D reconstruction a Pleuritis calcarea found in CT-thorax as well as the positions of pacemaker leads can be illustrated easily for teaching purposes.

and retroperitoneal organs in embalmed cadavers due to severe artifacts. These do not even allow the standard measurements of organ size. The commonly used embalming fluid, containing small amounts of formalin, not only causes a significant swelling of soft tissues $[4,8]$, but also produces artifacts, that severely impair image quality [9]. The major problem seems to be the production of gas, which has been described by Saranteas et al. [16] and Tsui et al. [17]. On the other hand, de Maeseneer et al. [18] showed that ultrasound quality of muscles of the hand in embalmed body donors was still better than ultrasound images of fresh cadavers due to a homogenising effect of the embalming fluid. Benkhadra et al. [14] showed similar results for imaging muscles, nerves and organs of the cervical region and ultrasound images

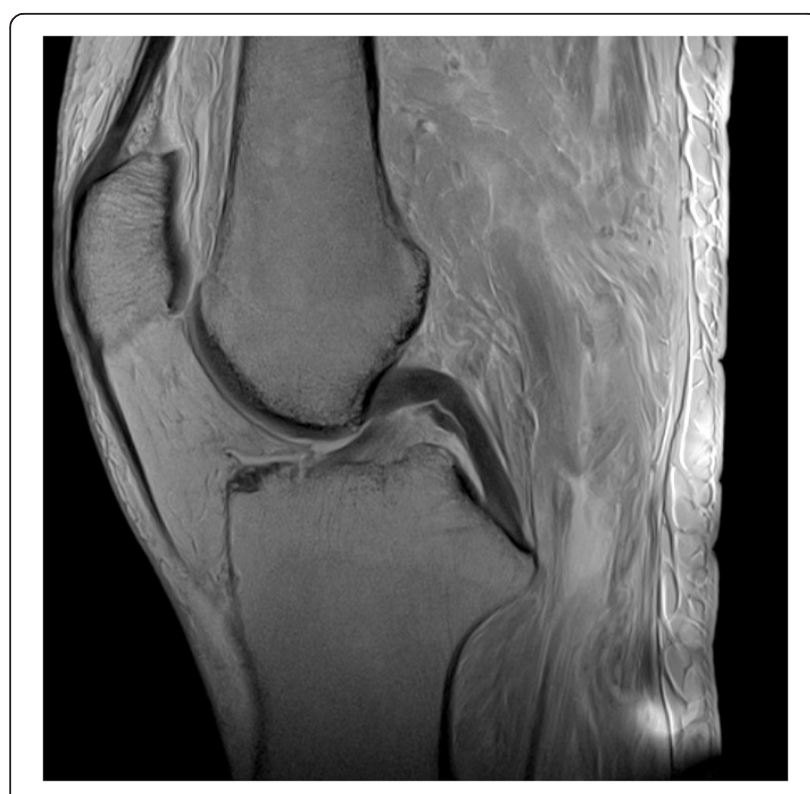

Figure 4 Very good quality of sagittal MRI image of the knee joint, skeletal and soft tissue structures are easily identifiable. of nerves and vessels of the lower extremity in embalmed cadavers even present images similar in quality to those taken from the living [19]. Further studies should investigate if those images offer useful information for teaching in the dissection course. Summing up, despite the theoretical advantage of this technique the rather poor imaging quality of intraabdominal and retroperitoneal organs disable the use of ultrasound in embalmed bodies. Based on the results of our study so far, ultrasound is not reasonable to be introduced to the dissection course as desired. Nevertheless, it remains the imaging tool of choice for 'living anatomy' courses on life subjects.

\section{Radiography}

Plain $\mathrm{x}$-ray techniques are preferred for a quick overview of skeletal structures as well as fluid accumulation in the lungs and distribution of gas content in the gut. In embalmed bodies this technique has a very limited use, even though mobile $x$-ray machines would allow imaging of human body donors right in the anatomical institute. In addition to severe gas artifacts, $x$-ray imaging was limited by the sagging of abdominal organs towards the spinal column due to the position of the body donors during fixation and storage. This prohibited the penetration of

Table 5 Evaluation of 3D reconstruction and feasibility of imaging techniques

\begin{tabular}{lcccc}
\hline & US & X-ray & CT & MRI \\
\hline 3D reconstruction possible & 0 & 0 & 2 & 1 \\
Price & 2 & 2 & 0 & 0 \\
Logistics & 2 & 1 & 0 & 0 \\
Overall feasibility & 2 & 1 & 0 & 0 \\
$\quad$ Total raw score & 6 & 4 & 2 & 1 \\
Calculated score & 1.5 & 1 & 0.5 & 0.25 \\
\hline
\end{tabular}

Rating: 2 = excellent, 1 = good, $0=$ poorly; for price: 2 = low expense, $0=$ high expense. 
radiation through the whole body. Our results are in contrast to the report of De Crop et al. [13], who found thorax images of embalmed cadavers to be equivalent to those of patients. This might be due to the difference in embalming solution and/or procedure. Nevertheless, radiography can be useful for upper and lower limb and joint imaging of embalmed human bodies. This needs to be further investigated.

\section{Computed tomography}

The quality of computed tomography (CT) images was slightly impaired by gas artifacts as previously described $[9,15]$. Due to perimortal changes and fixation artifacts, the lungs and other parenchymal organs were viewable on CT scans, but the precise detection of organ details was difficult. In the present study skeletal structures could clearly be detected, enabling CT imaging to be a suitable method to teach students and to identify pathological changes of the skeletal system.

\section{MRI}

The homogeneous appearance of embalmed tissues seems to be the disadvantage for magnetic resonance imaging (MRI). The fixation affects the water mobility and signal intensities of tissues, thus making the discrimination of tissues difficult $[8,19,20]$. Nevertheless, the authors declare, that for teaching purposes the image quality is still good enough because the topographical anatomy is still clearly observable $[4,8]$. In this study, the images of shoulder and knee joints were of very good quality.

\section{Conclusions}

We conclude that CT and MRI are suitable methods for imaging embalmed human bodies. In our view, the high logistic effort for those two techniques is justified given the superior image quality in comparison to ultrasound and plain $\mathrm{x}$-ray. Moreover, while ultrasound requires a skilled examiner and a constant visual feedback guiding the position of the ultrasound probe, topography of CT scans and MRIs are easier to grasp. Moreover, both CT and MRI even allow the three-dimensional reconstruction of complex anatomical structures or pathological processes. Despite the involved cost and effort we are convinced that the preparation of structures that underwent imaging just prior to anatomical dissection causes a great gain in knowledge and represents a valuable addition to early medical education.

Future studies need to clarify if the logistic and human investment into new teaching tools is well perceived and evaluated.

\section{Limitations}

This study only included a small number of human cadavers. For further studies we suggest the investigation of a larger sample of body donors, especially to identify possible gender differences. In this study we focused on the comparison of the feasibility of imaging techniques in embalmed cadavers for teaching purposes. The results reflect an only small part of possible scientific questions. For future studies a wider variety of aims and objectives, e.g. the comparison of sensitivity and specificity of the different imaging techniques for certain measurements, should be investigated to maximise the information of collected data. Furthermore, to accurately compare the image quality across all modalities, all four imaging techniques should be conducted with all body donors. We also recommend the comparison of different embalming techniques to see if they influence the image quality of the four modalities in different ways.

\section{Additional file}

Additional file 1: Intravascular gas artifacts in the arcuate arteries and in the surrounding tissue of the kidney severely limit the use of ultrasound in embalmed human cadavers. Movement of air bubbles can be seen in several small renal blood vessels, which did not dissolve by tissue compression with the ultrasound transducer.

\section{Competing interests}

The authors declare that they have no competing interests.

\section{Authors' contributions}

RS participated in the design of the study, coordinated the study procedure, participated in the interpretation of data, and drafted the manuscript. DS carried out the $x$-ray, $C T$, and MRI imaging, participated in the interpretation of data, and revised the manuscript critically. AR carried out the ultrasound imaging, participated in the interpretation of data, and revised the manuscript critically. JK participated in the design of the study, participated in the interpretation of data, and drafted the manuscript. $\mathrm{MH}$ participated in the design of the study, carried out the ultrasound imaging, participated in the interpretation of data, and revised the manuscript critically. HK participated in the design of the study, coordinated the study procedure, participated in the interpretation of data, and revised the manuscript critically. All authors read and approved the final manuscript.

\section{Authors' information}

RS, M.D., is a resident physician at the Department of Anatomy and Cell Biology, Faculty of Medicine, Martin-Luther-University Halle-Wittenberg, Halle (Saale), Germany. She teaches gross anatomy, neuroanatomy, embryology, and histology to first and second year medical students.

DS, M.D., is a senior physician at the Department of Radiology, Faculty of Medicine, Martin-Luther-University Halle-Wittenberg, Halle (Saale), Germany. He teaches radiology to third year medical students. Furthermore he is the head of the Dorothea Erxleben SkillsLab of the Faculty of Medicine, MartinLuther-University Halle-Wittenberg, Halle (Saale), Germany.

AR, M.D., is a senior physician at the Department of Internal Medicine, Division of Nephrology and Hypertension, Medical School Hannover, Germany. He teaches internal medicine, physical examination and nephrology at different time points during the model medical curriculum HannibaL (Hanoverian integrated, job-oriented and adaptive curriculum) at Hannover Medical School, as well as ultrasound techniques to residents and fellows.

JK, M.D., is an associate professor at the Department of Internal Medicine, Division of Nephrology and Hypertension, Medical School Hannover, Germany. He teaches internal medicine, physical examination and nephrology at different time points during the model medical curriculum HannibaL (Hanoverian integrated, job-oriented and adaptive curriculum) at Hannover Medical School. 
MH, M.D., is a senior physician at the Department of Internal Medicine, Division of Nephrology and Hypertension, Medical School Hannover, Germany. He teaches internal medicine, physical examination and nephrology at different time points during the model medical curriculum HannibaL (Hanoverian integrated, job-oriented and adaptive curriculum) at Hannover Medical School, as well as ultrasound techniques to residents and fellows.

HK, M.D., is a professor at the Department of Anatomy and Cell Biology, Faculty of Medicine, Martin-Luther-University Halle-Wittenberg, Halle (Saale), Germany. She teaches gross anatomy, embryology, and histology to first and second year medical students.

\section{Acknowledgments}

The authors thank Jana Gräfenhain, Julia Hallasch and Hans-Joachim Heine, anatomical preparators at the Department of Anatomy and Cell Biology, Faculty of Medicine, Martin-Luther-University Halle-Wittenberg, Halle (Saale), for their excellent assistance at all times.

\section{Author details}

'Department of Anatomy and Cell Biology, Martin Luther University of HalleWittenberg, Magdeburger Str. 8, 06108 Halle (Saale), Germany. ${ }^{2}$ Diagnostic Radiology, Faculty of Medicine, Martin Luther University of Halle-Wittenberg, Magdeburger Str. 8, 06108 Halle (Saale), Germany. ${ }^{3}$ Department of Internal Medicine, Division of Nephrology and Hypertension, Medical School Hannover, Carl-Neuberg-Str. 1, 30625 Hannover, Germany.

Received: 12 August 2013 Accepted: 21 October 2013

Published: 25 October 2013

\section{References}

1. McLachlan JC, Patten D: Anatomy teaching: ghosts of the past, present and future. Med Educ 2006, 40:243-253.

2. Pabst R, Westermann J, Lippert H: Integration of clinical problems in teaching gross anatomy: living anatomy, X-ray anatomy, patient presentations, and films depicting clinical problems. Anat Rec 1986, 215:92-94.

3. McLachlan JC: New path for teaching anatomy: living anatomy and medical imaging vs. dissection. Anat Rec B New Anat 2004, 281:4-5.

4. Entius CAC, van Rijn RR, Zwamborn AW, Kleinrensink GJ, Robben SGF: Influence of formaldehyde/ phenol fixation on MRI of the stifle joint and correlation with plastinated slices. J Int Soc Plastination 2004, 19:26-32.

5. Erkonen WE, Albanese MA, Smith WL, Pantazis NJ: Gross anatomy instruction with diagnostic images. Invest Radiol 1990, 25:292-294.

6. Erkonen WE, Albanese MA, Smith WL, Pantazis NJ: Effectiveness of teaching radiologic image interpretation in gross anatomy. A long-term follow-up. Invest Radiol 1992, 27:264-266.

7. Erkonen WE, Krachmer M, Cassell MD, Albanese MA, Stanford W: Cardiac anatomy instruction by ultrafast computed tomography versus cadaver dissection. Invest Radiol 1992, 27:744-747.

8. van der Made AD, Maas M, Beenen LFM, Oostra RJ, Kerkhoffs GMMJ: Postmortem imaging exposed: an aid in MR imaging of musculoskeletal structures. Skeletal Radiol 2012, 42:467-472.

9. Chew FS, Relyea-Chew A, Ochoa ER, JR: Postmortem computed tomography of cadavers embalmed for use in teaching gross anatomy. J Comput Assist Tomogr 2006, 30:949-954.

10. American Institute of Ultrasound in Medicine: AlUM Practice Guideline for the Performance of an Ultrasound Examination of the Abdomen and/ or Retroperitoneum. 2012.

11. European Commission: European Guidelines on Quality Criteria for Diagnostic Radiographic Images. 1996. EUR 16260 EN.

12. European Commission: European Guidelines on Quality Criteria for Computed Tomography. 1997. EUR 16262 EN.

13. De Crop A, Bacher K, van Hoof T, Smeets PV, Smet BS, Vergauwen M, Kiendys U, Duyck P, Verstraete K, D'Herde K, Thierens H: Correlation of contrast-detail analysis and clinical image quality assessment in chest radiography with a human cadaver study. Radiology 2012, 262:298-304.

14. Benkhadra M, Faust A, Ladoire S, Trost O, Trouilloud P, Girard C, Anderhuber F, Feigl G: Comparison of fresh and Thiel's embalmed cadavers according to the suitability for ultrasound-guided regional anesthesia of the cervical region. Surg Radiol Anat 2009, 31:531-535.
15. Bohl M, Francois W, Gest T: Self-guided clinical cases for medical students based on postmortem CT scans of cadavers. Clin Anat 2011, 24:655-663.

16. Saranteas T, Kostopanagiotou G, Paraskeuopoulos T, Vamvasakis E, Chantzi C, Anagnostopoulou S: Ultrasound examination of the sciatic nerve at two different locations in the lateral thigh: a new approach of identification validated by anatomic preparation. Acta Anaesthesio/ Scand 2007, 51:780-781.

17. Tsui BCH, Dillane D, Pillay J, Ramji A, Walji AH: Cadaveric ultrasound imaging for training in ultrasound-guided peripheral nerve blocks: lower extremity. Can J Anaesth 2007, 54:475-480.

18. De Maeseneer M, Jager T, Vanderdood K, van Roy P, Shahabpour M, Marcelis S: Ultrasound during dissection of cadaveric specimens: a new method for obtaining ultrasound-anatomic correlations in musculoskeletal radiology. Eur Radiol 2004, 14:870-874.

19. Dashner RA, Chakeres DW, Kangarlu A, Schmalbrock P, Christoforidis GA, DePhilip RM: MR imaging visualization of the cerebral microvasculature: a comparison of live and postmortem studies at $8 \mathrm{~T}$. AJNR Am J Neuroradiol 2003, 24:1881-1884.

20. Pfefferbaum A, Sullivan EV, Adalsteinsson E, Garrick T, Harper C: Postmortem MR imaging of formalin-fixed human brain. Neuroimage 2004, 21:1585-1595.

\section{doi:10.1186/1472-6920-13-143}

Cite this article as: Schramek et al:: Imaging in anatomy: a comparison of imaging techniques in embalmed human cadavers. BMC Medical Education 2013 13:143.

\section{Submit your next manuscript to BioMed Central and take full advantage of:}

- Convenient online submission

- Thorough peer review

- No space constraints or color figure charges

- Immediate publication on acceptance

- Inclusion in PubMed, CAS, Scopus and Google Scholar

- Research which is freely available for redistribution

Submit your manuscript at www.biomedcentral.com/submit
Ciomed Central 